\title{
Warfarin involvement, in comparison to NOACs, in the development of systemic atherosclerosis
}

\author{
Romeo Gabriel Mihaila
}

Adverse effects of drugs are one of the objective criteria used for choosing the most appropriate anticoagulant. It is worrying that warfarin may be involved in the progression of systemic atherosclerosis, as more and more articles suggest. Warfarin has been widely used in the past and has greater efficacy compared to dabigatran in patients with mechanical heart valves; there is an antidote to it and it is cheap. Unfortunately, warfarin inhibits the synthesis and activity of Matrix-Gla-Protein, which is the major vitamin K-dependent inhibitor of arterial calcification - an active process associated with atherosclerosis, stimulated by inflammatory mechanisms. Vitamin K antagonizes the NF-KB signaling mechanism and contributes to the prevention of arterial calcifications. Warfarin given in experimental animal models of atherosclerosis contributed to the occurrence of an increased number of aortic calcifications. Warfarin treatment used in clinical trials was associated with the progressive increase of coronary atheroma calcification. Younger patients are more sensitive to warfarin-related arterial calcifications compared to older patients, due to warfarin-induced cellular senescence changes. Non-vitamin $\mathrm{K}$ antagonist direct oral anticoagulants do not interact with vitamin $\mathrm{K}$. Edoxaban reduces the inflammatory process in the vascular walls and the proliferation of smooth vascular muscle cells, so it is involved in the prevention of vascular maladaptive remodeling process. Apixaban is able to stabilize the coronary atherosclerotic process. Randomized clinical trials are needed to evaluate the impact of warfarin on plaque stability and cardiovascular evolution of patients.

Key words: atherosclerosis, direct oral anticoagulants, thrombosis, vascular calcification, warfarin

Received: October 31, 2021; Revised: February 7, 2022; Accepted: February 8, 2022; Available online: February 18, 2022 https://doi.org/10.5507/bp.2022.008

(c) 2022 The Authors; https://creativecommons.org/licenses/by/4.0/

Faculty of Medicine, "Lucian Blaga" University of Sibiu, Romania

Corresponding author: Romeo Gabriel Mihaila, e-mail: romeomihaila@yahoo.com

\section{INTRODUCTION}

Today, venous thromboembolism is considered to be the third most common cause of vascular death. Anticoagulation therapy is indispensable for its treatment, but despite this therapy, up to half of patients with deep vein thrombosis develop postthrombotic syndrome. If in the past antivitamins $\mathrm{K}$ were the main anticoagulant drugs for long-term therapy, today, the range of available products has expanded. Direct oral anticoagulants do not require monitoring of the international normalized ratio (INR) and are safer than warfarin, but bleeding is the major side effect, especially in cancer patients ${ }^{1}$. One of the advantages of warfarin is its greater efficacy compared to dabigatran in patients with mechanical heart valves ${ }^{2}$. Warfarin suppresses thrombin generation induced by the presence of mechanical heart valves. But mechanical heart valves are involved in generating higher concentrations of factor Xa than those that can be controlled with rivaroxaban or apixaban. Recently published are the results of a study claiming that the combination of rivaroxaban and dabigatran is more effective than either agent alone. It appears that concomitant inhibition of factor Xa and thrombin is superior to that of either clotting enzyme alone ${ }^{2}$. The scientific community agrees that safer anticoagulants are needed. New drugs are being developed to enhance fibrinolysis and decrease inflammation and anticoagulant products against the factor XII and factor XI (ref. ${ }^{1}$ ).

Vitamin $\mathrm{K}$ is fat-soluble and is involved in the activation of various vitamin K-dependent proteins. A deficit of vitamin $\mathrm{K}$ is involved in the pathogenesis of cardiovascular disease ${ }^{3}$. Gamma-carboxylation is needed for activation of vitamin $\mathrm{K}$ and other proteins which are involved in the inhibition of vascular calcification and bone formation ${ }^{4}$. In recent years it has been shown that warfarin can increase systemic vascular calcification ${ }^{5,6}$ by inhibiting the carboxylation of Matrix-Gla-Protein (MGP) ( ref. $^{7}$ ). While anti-vitamin $\mathrm{K}$ products are involved in the onset and progression of vascular calcifications, oral direct factor Xa inhibitors, such as endoxaban are able to reduce the inflammatory process in the vascular walls and the proliferation of smooth vascular muscle cells. Thus, they are involved in the prevention of the vascular maladaptive remodeling process ${ }^{8}$. Apixaban is another oral direct factor Xa inhibitor, which does not interact with vitamin $\mathrm{K}$ $\left(\right.$ ref. $\left.^{5}\right)$, like endoxaban. It has been shown to be superior to warfarin at preventing stroke and systemic embolism and it is able to stabilize the coronary atherosclerotic process 9 . The patients with non-valvular atrial fibrillation treated with warfarin had higher total, calcified and low attenuation plaque volume compared to those treated with apixaban ${ }^{5}$. 


\section{CALCIFICATIONS OF ATHEROMATOUS PLAQUES}

The following processes take place during atherogenesis: an invasion of cells involved in inflammation, the formation of lipid deposits and even fatty plaques which allow accumulation of minerals, including calcium. All of this disrupts the vascular endothelium and its regulatory role in arterial function ${ }^{4}$.

Coronary artery calcification is no longer considered today to be a passive, degenerative, and quiescent process, but an active process associated with atherosclerosis, stimulated by inflammatory mechanisms. Calcification occurs within the intimal and medial layers of the vessel wall, and the pathway is similar to that of bone formation ${ }^{10}$. Osteoblast-like cells that accumulate in the muscular layer of arteries are involved in the vascular calcification ${ }^{4}$. Macrophages in the intimal and medial layers of arterial walls contribute to osteoblastic differentiation of vascular smooth muscle cells, through the release of proinflammatory cytokines (e.g. IL-1 $\beta$, IL- 6 , TNF- $\alpha$ ), and oncostatin $\mathrm{M}$. The result is a vascular inflammatory process NF-kBmediated and the appearance of vascular calcifications ${ }^{3}$. Various lesions can be observed in coronary artery disease, from early microcalcifications to well-developed calcified fibroatheromas. There are gender and race differences regarding the prevalence and development of coronary artery calcification ${ }^{10}$.

Vascular calcification is frequently observed in patients with atherosclerosis and diabetes ${ }^{11}$. Indeed, calcification is a defining aspect of advanced atherosclerosis. It has been established that proteoglycan 4 (PRG4) is a key molecular signature for calcified plaques. It can be found in the smooth muscle cell early, when the intima is only thickened, and in the extracellular matrix, surrounding the large calcifications present in the advanced stages of atherosclerosis. PRG4 is involved in the modulation of smooth muscle cell function and osteogenic phenotype and responds to TGFb1 signaling, SMAD3 and SOX9 activation. This argues for its role in the process of atherosclerotic plaque stability ${ }^{12}$.

Detection and monitoring of the progression of atherosclerotic plaques can be performed using an ApoE -/- mouse model and an adapted positron emission tomography / computed tomography device. Sodium [18F] Fluoride is known to have high specificity for calcifications located in the vascular walls. Thus, this device allowed the detection of spotty calcifications in the proximal aorta; the highest uptake of the contrast substance was in the aortic arch and left ventricle of animals that received warfarin ${ }^{13}$.

\section{THE EFFECT OF WARFARIN ON ATHEROMATOUS PLAQUE CALCIFICATIONS}

In vitro and in vivo studies have established that vitamin $\mathrm{K}$ antagonizes the NF-kB signaling mechanism. Thus, it fights against inflammation and, implicitly, contributes to the prevention of arterial calcifications. Vitamin $\mathrm{K}$ is also involved in preventing the progression of arterial calcifications by increasing the activity of the MGP - known to be the major inhibitor of calcification in the soft tissue. One of the inactive forms of the protein - dephospho-uncarboxylated MGP may reflect vitamin K deficiency and may be a very useful biomarker of vascular calcification and cardiovascular disease ${ }^{3}$.

More and more articles mention the pro-calcifying effect of vitamin $\mathrm{K}$ antagonists, including warfarin, on the vessel wall ${ }^{9,10}$. Indeed, long-term use of warfarin, but not of the non-vitamin $\mathrm{K}$ antagonist direct oral anticoagulants (NOACs), can have as a side effect increase in the risk of vascular calcification or even induce such calcifications in some patients ${ }^{4}$.

It is known that warfarin inhibits the synthesis and activity of MGP, which is therefore a vitamin K-dependent inhibitor of arterial calcification ${ }^{14}$.

The warfarin diet given to rats contributed to the occurrence of an increased number of aortic calcifications. But the distribution and severity of calcification was significantly lower in animals treated with $\beta$-aminopropionitrile, a lysyl oxidase inhibitor. Lysyl oxidase is known to be an enzyme involved in cross-linking collagen. This result claims that collagen metabolism has an important role in the early pathogenesis of calcifications in the arterial walls ${ }^{15}$.

The combination of warfarin and ginseng, may favor the appearance of thrombosis and the progression of atherosclerosis. In vitro it was observed that the combined treatment with warfarin and ginsenosides increased the adhesion ability between platelets and endothelial cells and matrigel. In addition, ginsenosides were able to decrease the anticoagulation effect of warfarin in experimental model of hyperlipemic rats, due to the increased expression of plasma levels of factors invloved in coagulation and hepatic cytochrome P450 enzymes after long-term treatment with warfarin in combination with ginsenoids ${ }^{16}$.

\section{CHALLENGES OF ANTICOAGULANT TREATMENT}

Anticoagulant treatment is the most effective way to prevent both arterial and venous thromboembolism ${ }^{17}$.

\section{Benefits and disadvantages of warfarin}

Warfarin treatment requires INR monitoring, but the absence of a dose does not cause significant changes in protrombin time. Vitamin K (the antidote to warfarin) can be given in case of overdose. The fact that warfarin is cheap is an important advantage.

Patients treated with warfarin should not take vitamin $\mathrm{K}$. Vitamin $\mathrm{K}$ is a cofactor involved in the $\gamma$-carboxylation of vitamin K-dependent proteins such as: coagulation factors, MGP (which inhibits the soft tissue calcification in the vessel wall), osteocalcin (necessary for bone matrix formation), and the growth arrest-specific 6 (with antiapoptotic effect on vascular smooth muscle cells). Vitamin $\mathrm{K}$ deficiency is involved in the pathogenesis of 
osteoporosis, vascular calcification, and inhibits vessel angiogenesis ${ }^{18}$. It should also be noted that warfarin (and heparin) is involved in the occurrence of acute peripheral and / or cutaneous ischemic syndrome, through thrombotic injuries that occur locally ${ }^{19}$.

\section{Benefits and disadvantages of oral antagonist anticoagulants}

The following NOACs are currently available: dabigatran (a thrombin inhibitor), and apixaban, betrixaban, edoxaban and rivaroxaban (factor Xa inhibitors) (ref. ${ }^{18,20}$ ). They are increasingly used for the prophylaxis of thrombotic stroke risk in many patients with atrial fibrillation ${ }^{18}$, but also in other categories of patients, for the prophylaxis and treatment of various thrombosis. NOACs do not require INR monitoring, but the absence of a dose leaves patients unprotected from a possible thrombotic episode. There is an antidote only against dabigatran (idarucizumab), but it is expensive and not available in any medical service.

The use of NOACs (rivaroxaban, dabigatran, edoxaban) in lower doses has been shown to provide protection against all-cause mortality in patients with atherosclerosis with cardiac localization or peripheral artery disease ${ }^{21}$.

NOACs were at least as effective with warfarin for stroke prevention in patients with atrial fibrillation and for venous thromboembolism therapy, but they produced less intracranial bleeding episodes ${ }^{20}$. Indeed, they are able to inhibit intracranial hemorrhage, but also stroke, and even death compared to warfarin ${ }^{18}$. One of the advantages of administering NOACs is that they can be given in fixed doses, without routine INR monitoring. It is established that NOACs have a favorable risk / benefit ratio compared to warfarin. The use of NOACs depends on the results of individual trials, the presence and degree of renal dysfunction, the potential for interaction with other drugs, and preference for once- or twice-daily dosing ${ }^{20}$.

\section{Comparisons between warfarin and NOACs}

An analysis was made of the net clinical benefit of 7963 adult patients with symptomatic proximal deep vein thrombosis and / or pulmonary embolism treated with dabigatran etexilate (administered at a dose of $150 \mathrm{mg}$ twice daily) compared to warfarin. If the definition of the net clinical benefit took into account major bleeding events and clinically relevant non-major hemorrhagic events, it was in favor of dabigatran etexilate, independent of the quality of INR control ${ }^{22}$.

Another study compared warfarin with dabigatran etexilate given for the prophylaxis and treatment of venous thromboembolism in thrombophilic patients or in those with antiphospholipid antibody syndrome. Rates of bleeding events were lower in the dabigatran etexilate group than in the warfarin group ${ }^{23}$.

\section{The anticoagulant treatment in older patients}

Prophylaxis and treatment of thromboembolism in older individuals is not easy, due to the high risk of both thrombosis and bleeding, favored by advanced age, comor- bidities, and the interaction of anticoagulant drugs with others that they use frequently ${ }^{17}$.

Warfarin and NOACs are not used enough and are often underdosed in stroke prevention in older patients with atrial fibrillation due to concerns about the risk of bleeding. In addition, the compliance of elderly patients with anticoagulant therapy is lower than that of younger patients ${ }^{17}$.

It is recommended that the anticoagulant treatment be customized regarding the type of anticoagulant suitable for a particular patient and regarding the appropriate $\operatorname{dose}^{20}$.

\section{EXPERIMENTAL STUDIES}

More and more studies establish that warfarin promotes the appearance and progression of arterial calcifications ${ }^{24}$.

A rat model of arterial calcification can be obtained by a combination of warfarin and vitamin $\mathrm{K} 1$ ( ref. $^{11}$ ).

Warfarin induced calcific valve degeneration in an experimental mouse model of atherosclerosis. Neither warfarin nor rivaroxaban contributed to the progression of atherosclerosis. In contrast, rivaroxaban was not able to prevent the osteogenic conversion of cells, but decreased the over-expression of COX-2 produced by inflammatory mediators, demonstrating that it has a better safety profile on the risk of arterial disease progression ${ }^{7}$.

Warfarin administered for 6 weeks to mice fed a Western-type diet contributed to the formation and progression of atherosclerotic lesions that had a pro-inflammatory phenotype. Its administration for 12 weeks in the same experimental model stimulated the formation and activity of plaque calcification. On the contrary, dabigatran contributed both to the reduction of the progression of atherosclerotic plaques and to the presence and activity of calcifications in these plaques compared to the animals in the control group. In vitro, warfarin and thrombin produced an increase in oxidative stress in smooth muscle cells present in blood vessel walls and extracellular vesicle release; dabigatran prevented these processes ${ }^{25}$.

\section{CLINICAL STUDIES}

A study that included 6,655 participants found that patients treated with warfarin had calcified coronary plaques much more frequently than those untreated with anti-vitamin $\mathrm{K}$. The score of arterial calcifications increased with increasing serum levels of interleukin-6 and tumor necrosis factor alpha. Warfarin contributed to the appearance of deteriorated calcification in young vascular smooth muscle cells present in the early stage of calcification, according to one in vitro study. In the human study it was found that younger patients are more sensitive to warfarinrelated arterial calcifications compared to older patients, due to warfarin-induced cellular senescence changes ${ }^{24}$.

A recent analysis included 8 prospective randomized trials with patients diagnosed with coronary artery 
disease, who were monitored by coronary intravascular ultrasound. It was found that warfarin treatment was associated with the progressive increase of coronary atheroma calcification, without significant changes in atheroma volume. Further studies are needed to establish the impact that these changes have on plaque stability and cardiovascular evolution ${ }^{14}$.

A pilot study included patients with chronic atrial fibrillation, treated for at least 12 months with warfarin, who switched to anticoagulant therapy with rivaroxaban for at least 6 months. It was observed that this change led to increased serum levels of bone alkaline phosphatase and decreased serum levels of under-carboxylated osteocalcin. In addition, serum level of osteopontin (a biomarker of atherosclerosis and osteoporosis), pulse wave velocity, and augmentation index decreased under rivaroxaban treatment ${ }^{18}$.

\section{CONCLUSIONS}

Vitamin K deficiency is involved in the pathogenesis of osteoporosis, vascular calcification, and inhibits vessel angiogenesis ${ }^{18}$. Coronary artery calcification is an active process associated with atherosclerosis, stimulated by inflammatory mechanisms ${ }^{10}$.

Warfarin is a cheap treatment, that requires INR monitoring, but the absence of a dose does not cause significant changes in prothrombin time. Vitamin K (the antidote to warfarin) can be given in case of overdose. Warfarin is involved in the acute peripheral and / or cutaneous ischemic syndrome ${ }^{19}$.

Warfarin inhibits the synthesis and activity of MGP, which is a vitamin K-dependent inhibitor of arterial calcification ${ }^{14}$. Long-term use of warfarin, but not of the NOACs, can increase in the risk of vascular calcification or even induce such calcifications in some patients.

NOACs can be given in fixed doses, without routine INR monitoring. It was established that NOACs have a favorable risk / benefit ratio compared to warfarin ${ }^{20}$. Edoxaban reduces the inflammatory process in the vascular walls and the proliferation of smooth vascular muscle cells, and is involved in the prevention of vascular maladaptive remodeling process. Apixaban is able to stabilize the coronary atherosclerotic process ${ }^{9}$.

Before recommending another anticoagulant, randomized clinical trials are needed to evaluate the impact of warfarin on plaque stability and cardiovascular evolution.

\section{ABBREVIATIONS}

INR, international normalized ratio; MGP, Matrix-GlaProtein; NOACs, non-vitamin K antagonist direct oral anticoagulants; PRG4, proteoglycan 4.

\section{Search strategy and selection criteria}

Strategy research aimed at analyzing recent data on the pathogenetic mechanism of atheromatous plaque formation and progression and the role of warfarin in this pro- cess. Scientific articles published in the last five years were searched using PubMed and the Web of Science databases. All searches were up to date as of September 30, 2021. The search terms used were: "warfarin" and "atherosclerosis". Only English language papers were reviewed.

Conflict of interest statement: The author declares that there are no conflicts of interest regarding the publication of this article.

\section{REFERENCES}

1. Weitz Jl, Chan NC. Novel antithrombotic strategies for treatment of venous thromboembolism. Blood 2020;135(5):351-9.

2. Jaffer IH, Fredenburgh JC, Stafford A, Whitlock RP, Weitz JI. Rivaroxaban and Dabigatran for Suppression of Mechanical Heart Valve-Induced Thrombin Generation. Ann Thorac Surg 2020;110(2):582-90.

3. Shioi A, Morioka T, Shoji T, Emoto M. The Inhibitory Roles of Vitamin K in Progression of Vascular Calcification. Nutrients 2020;12(2):583.

4. Siltari A, Vapaatalo H. Vascular Calcification, Vitamin K and Warfarin Therapy - Possible or Plausible Connection? Basic Clin Pharmacol Toxicol 2018;122(1):19-24.

5. Win TT, Nakanishi R, Osawa K, Li D, Susaria SS, Jayawardena E, Hamal S, Kim M, Broersen A, Kitslaar PH, Dailing C, Budoff MJ. Apixaban versus warfarin in evaluation of progression of atherosclerotic and calcified plaques (prospective randomized trial). Am Heart J 2019;212:129-33.

6. Lee J, Nakanishi R, Li D, Shaikh K, Shekar C, Osawa K, Nezarat N, Jayawardena E, Blanco M, Chen M, Sieckert M, Nelson E, Billingsley D, Hamal S, Budoff MJ. Randomized trial of rivaroxaban versus warfarin in the evaluation of progression of coronary atherosclerosis. Am Heart J 2018;206:127-30.

7. Rattazzi M, Faggin E, Bertacco E, Nardin C, Pagliani L, Plebani M, Cinetto F, Guidolin D, Puato M, Pauletto P. Warfarin, but not rivaroxaban, promotes the calcification of the aortic valve in ApoE-/- mice. Cardiovasc Ther 2018;36(4):e12438.

8. Millenaar D, Bachmann P, Böhm M, Custodis F, Schirmer SH. Effects of edoxaban and warfarin on vascular remodeling: Atherosclerotic plaque progression and collateral artery growth. Vascul Pharmacol 2020;127:106661.

9. Osawa K, Nakanishi R, Win TT, Li D, Rahmani S, Nezarat N, Sheidaee $\mathrm{N}$, Budoff MJ. Rationale and design of a randomized trial of apixaban vs warfarin to evaluate atherosclerotic calcification and vulnerable plaque progression. Clin Cardiol 2017;40(10):807-13.

10. Andrews J, Psaltis PJ, Bartolo BAD, Nicholls SJ, Puri R. Coronary arterial calcification: A review of mechanisms, promoters and imaging. Trends Cardiovasc Med 2018;28(8):491-501.

11. Li M, Wang Z, Shao J, Li S, Xia H, Yu L, Hu Z. Captopril Attenuates the Upregulated Connexin 43 Expression in Artery Calcification. Arch Med Res 2020;51(3):215-23.

12. Seime $T$, Akbulut AC, Liljeqvist $M L$, Siika A, Jin $H$, Winski G, van Gorp RH, Karlöf E, Lengquist M, Buckler AJ, Kronqvist M, Waring OJ, Lindeman JHN, Biessen EAL, Maegdefessel L, Razuvaev A, Schurgers LJ, Hedin U, Matic L. Proteoglycan 4 Modulates Osteogenic Smooth Muscle Cell Differentiation during Vascular Remodeling and Intimal Calcification. Cells 2021;10(6):1276.

13. Florea A, Sigl JP, Morgenroth A, Vogg A, Sahnoun S, Winz OH, Bucerius J, Schurgers LJ, Mottaghy FM. Sodium [18F]Fluoride PET Can Efficiently Monitor In Vivo Atherosclerotic Plaque Calcification Progression and Treatment. Cells 2021;10(2):275.

14. Andrews J, Psaltis PJ, Bayturan O, Shao M, Stegman B, Elshazly M, Kapadia SR, Tuzcu EM, Nissen SE, Nicholls SJ, Puri R. Warfarin Use Is Associated With Progressive Coronary Arterial Calcification: Insights From Serial Intravascular Ultrasound. JACC Cardiovasc Imaging 2018;11(9):1315-23.

15. Uto K, Yoshizawa S, Aoki C, Nishikawa T, Oda H. Inhibition of extracellular matrix integrity attenuates the early phase of aortic medial calcification in a rodent model. Atherosclerosis 2021;319:10-20.

16. Lin JF, Fan LL, Li BW, Zhao RR, Jiang LG, Zhang BC, Lu YS, Shao JW. 
A study to evaluate herb-drug interaction underlying mechanisms: An investigation of ginsenosides attenuating the effect of warfarin on cardiovascular diseases. Eur J Pharm Sci 2020;142:105100.

17. Chan NC, Eikelboom JW. How I manage anticoagulant therapy in older individuals with atrial fibrillation or venous thromboembolism. Blood 2019;133(21):2269-78.

18. Namba S, Yamaoka-Tojo M, Kakizaki R, Nemoto T, Fujiyoshi K, Hashikata T, Kitasato L, Hashimoto T, Kameda R, Meguro K, Shimohama T, Tojo T, Ako J. Erratum to: Effects on bone metabolism markers and arterial stiffness by switching to rivaroxaban from warfarin in patients with atrial fibrillation. Heart Vessels 2017;32(8):983.

19. Santiago MB, Paz A. Acute Peripheral and/or Cutaneous Ischemic Syndrome: What Rheumatologists Should Know. J Clin Rheumato 2021;27(2):73-9.

20. De Caterina R, Ageno W, Agnelli G, Chan NC, Diener HC, Hylek E, Raskob GE, Siegal DM, Verheugt FWA, Lip GYH, Weitz J. The NonVitamin K Antagonist Oral Anticoagulants in Heart Disease: Section V-Special Situations. Thromb Haemost 2019;119(1):14-38.

21. Cappato R, Chiarito M, Giustozzi M, Briani M, Ali H, Riva L, Bonitta G, Lodigiani C, Furlanello F, Balla C, Lupo P, Stefanini G. Lower dose direct oral anticoagulants and improved survival: A combined anal- ysis in patients with established atherosclerosis. Eur J Intern Med 2021;83:14-20.

22. Feuring M, Schulman S, Eriksson H, Kakkar AJ, Schellong S, Hantel S, Schueler E, Kreuzer J, Goldhaber SZ. Net clinical benefit of dabigatran vs. warfarin in venous thromboembolism: analyses from RE-COVER ${ }^{\oplus}$, RE-COVER ${ }^{T M}$ II, and RE-MEDY ${ }^{T M}$. J Thromb Thrombolysis 2017;43(4):484-9.

23. Goldhaber SZ, Eriksson H, Kakkar A, Schellong S, Feuring M Fraessdorf M, Kreuzer J, Schueler E, Schulman S. Efficacy of dabigatran versus warfarin in patients with acute venous thromboembolism in the presence of thrombophilia: Findings from RE-COVER ${ }^{\oplus}$, RE-COVER ${ }^{\text {TM }}$ II, and RE-MEDYTM. Vasc Med 2016;21(6):506-14.

24. Wei N, Lu L, Zhang H, Gao M, Ghosh S, Liu Z, Qi J, Wang J, Chen J, Huang $\mathrm{H}$. Warfarin Accelerates Aortic Calcification by Upregulating Senescence-Associated Secretory Phenotype Maker Expression. Oxid Med Cell Longev 2020;2020:2043762.

25. van Gorp RH, Dijkgraaf I, Bröker V, Bauwens M, Leenders P, Jennen D, Dweck MR, Bucerius J, Briedé JJ, van Ryn J, Brandenburg V, Mottaghy F, Spronk HMH, Reutelingsperger CP, Schurgers LJ. Off-target effects of oral anticoagulants - vascular effects of vitamin K antagonist and non-vitamin $\mathrm{K}$ antagonist oral anticoagulant dabigatran etexilate. J Thromb Haemost 2021;19(5):1348-63. 